\title{
Vericut - Configuring CNC Systems for Turning Center with Sub-spindle
}

\author{
Tsvetan Kaldashev \\ Technical university of Sofia \\ Sofia, Bulgaria \\ kaldashev.cvetan@abv.bg
}

\begin{abstract}
This report discusses the configuration of some preparatory and auxiliary commands for the Fanuc 18i TB CNC system for a counter-spindle lathe using special macros called Vericut macros. In most cases, this is necessary because the Vericut library of machines and CNC systems does not have the specific machine and system or do not correspond to the actual ones, $i$. e. there is no match between them. The need to configure the CNC control system is related to the fact that it is possible to perform simulation, optimization and verification of control programs in a virtual environment before proceeding to the processing of workpieces on the real machine.
\end{abstract}

Keywords - Vericut, CNC, verification, turning center, subspindle

\section{INTRODUCTION}

To reduce the risks associated with the defect of the workpiece and reduce the cost of the final product, products are used in which this check can be performed in a virtual environment. When using such a class of products, the stages of technological preparation for processing the workpiece have the structure shown in fig. 1.

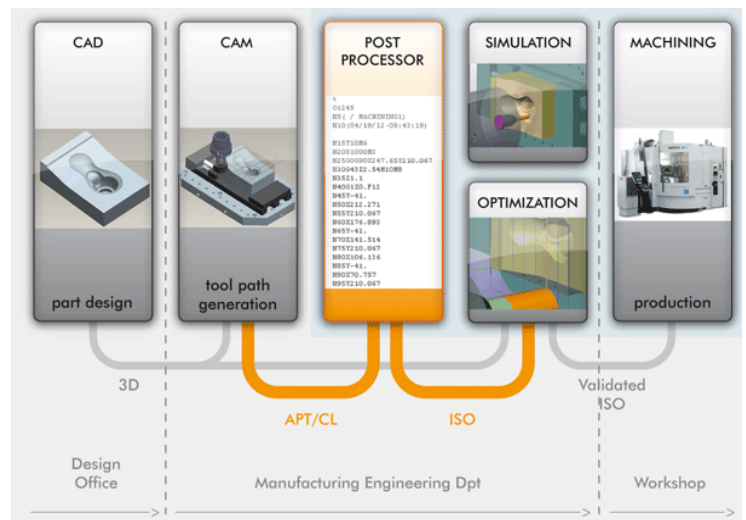

Fig. 1. Stages of technological preparation with the use of products for virtual simulation of the processing [2]
After the simulation stage, an evaluation of the result is performed, after which, based on the obtained result, in some cases optimization is required. Such products are VERICUT, NCSIMUL and others. VERICUT [1] is a product of high grade, that allows for verification and optimization of the tool path, checking for collisions, the simulation of cutting processes. All these possibilities are available for milling, turning, laser, wire erosion discharge machines, turning centers, multi-task machines and robots.

When verifying the tool path, a real machine, tools, holders, workpiece are used, and the simulation is by taking material in a virtual environment. Both the generated driver from the CAM package and the CL data file can be used to simulate the process.

\section{CONFIGURING A CNC SYSTEM FOR A TURNING CENTER WITH A SUB-SPINDLE}

To solve the problem, Vericut's capabilities for configuring the CNC system are used. In this case, CNC from the Vericut library will be used, where only the necessary M- and G-commands for the Emco Maxxturt 45 machine with Fanuc 18i TB will be configured (added). In fig. 2 shows the kinematic model of the machine [3].

As is well known, the manufacturer of CNC machines determines the purpose of $\mathrm{M}$-commands (other than those standardized by ISO) to control more specific actions. Here is considered the possibility of configuring the $\mathrm{M}$ commands related to the control of the main and counter spindle in the two characteristic modes for the lathe centers, namely the commands with which they are set in the operating mode when they realize the cutting speed, i.e. turning and mode in which they are used as a circular axis. Table 1 gives M-commands [6] with their meaning, which will be configured to control the machine. 

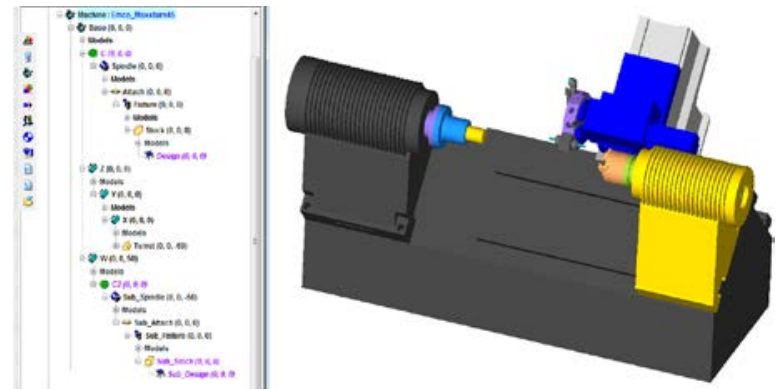

Fig. 2. Kinematic model of the EmcoMaxxturn 45 machine

In turning centers, the typical hole machining cycles have a difference from the machining cycles on drilling and milling machines and machining centers, consisting mainly in the preparation command and on which axis the machining is performed (X-axis or Z-axis). These commands will also be configured.

TABLE 1 M-COMMANDS TO BE CONFIGURED

\begin{tabular}{|c|l|}
\hline $\begin{array}{c}\text { M- } \\
\text { comm } \\
\text { ands }\end{array}$ & \multicolumn{1}{|c|}{ Function } \\
\hline M52 & Selection main spindle C-axis \\
\hline M53 & Deselection main spindle C-axis \\
\hline M69 & Open jaws of the main spindle \\
\hline M168 & Open jaws of the counter spindle \\
\hline M252 & Selection counter spindle C-axis \\
\hline M253 & Deselection counter spindle C-axis \\
\hline M203 & Counter spindle ON, clockwise rotation \\
\hline M204 & Counterspindle ON, counter-clockwise rotation \\
\hline M205 & Counter spindle OFF \\
\hline M303 & Driven tool ON, clockwise rotation \\
\hline M304 & Driven tool ON, counter-clockwise rotation \\
\hline M305 & Driven tool OFF \\
\hline
\end{tabular}

The commands used by the CNC are configured from the G-code Processing window using the so-called Vericut Macro [4]. In essence, these are "macros" developed by Vericut, where their use triggers a certain action. Auxiliary M-commands related to the process of transferring the workpiece between the two spindles are set in the Pick_Off section. This is how the settings for the M69 command are made: in the Add / Modify Word / Address window (fig. 3) the M-command is set. The MacroName uses the ClampCompName macro, which sets the gadget name in the Override Text field in this case Fixture. This field records the name of the device used in the kinematic model of the machine.

The state in which the device is located, set with the macro ClampCompName, ie. the jaws of the chuck (collet device) are open or closed, it is controlled by the macro ClampOnOff. The status is set to 0 off and 1 on. The mode is set in the Override Value field (fig. 3). The other Mcommands in the Pick_Off section are configured in a similar way, as for command M168 with macro
ClampCompName the name of the equipment of the opposite spindle is set - Sub_Fixture (fig. 3).

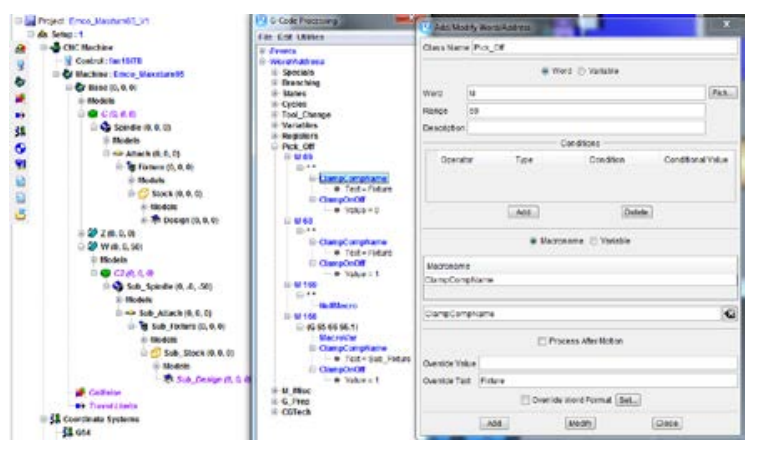

Fig. 3. Windows Add / Modify Word / Address setup commands CNC

In fig. 4 shows the structure and Vericut Macro used to configure the M52 command. After reading command M52, the CNC is able to use the main spindle as a circular axis $\mathrm{C} 1$.

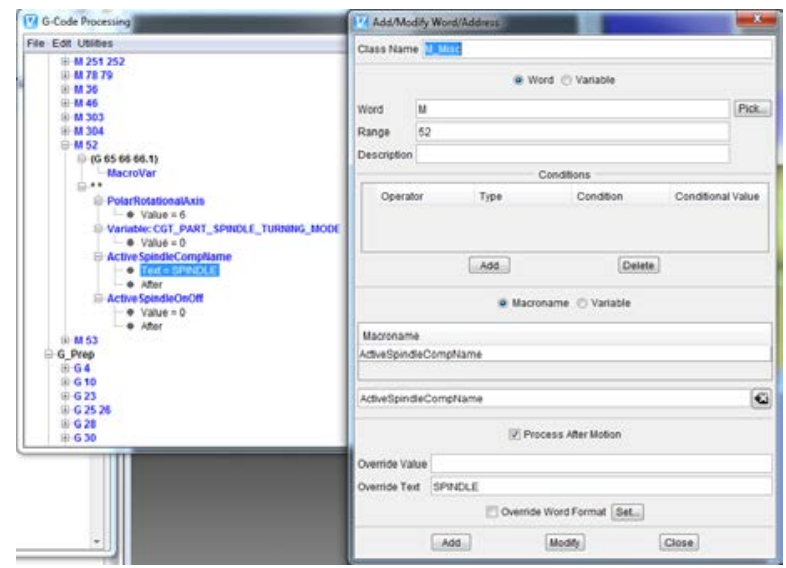

Fig. 4. 4 Structure of the M52 command with the Vericut Macro used to configure it

In practice, this means that the "active" position of the turret is used, i.e. instrumental transitions are made for processing of openings, shifted in relation to the axis of rotation of the workpiece, flat or profile sections are processed, etc. This is also related to the use of polar and cylindrical interpolation mode. Setting this mode is done with the PolarRotationalAxis macro.

In this mode, the axis is set, which is controlled in Cartesian coordinates. The setting is done with a number, where the correspondence number-controllable axis is observed, namely: 4-A, 5-B, 6-C, 10-A2, 11-B2, 12-C2. The variable CGT_PART_SPINDLE_TURNING_MODE is entered by the user who controls the circular axis mode. In this case it is the variable for the main spindle. A value of 0 deactivates the lathe mode and 1-activates the lathe mode. The macro ActiveSpindleCompName specifies which spindle (main or counter) will be set as a circular axis. When using the M52 command, it is necessary to stop the main rotational movement of the spindle. This is controlled with the ActiveSpindleOnOff macro, where the current state of the spindle is set in the Override Value field, with the M52 command running (0-off, 1-on). For commands M252 and M253 proceed in a similar way. To 
configure the commands, to control the direction of rotation of the "active" position of the turret of the machine - commands M303, M304, M305, the macro ActiveSpindleActiveTool is used, which activates the tool set in the "active" position of the turret. The direction of rotation of the tool set in the "active" position is done with the already mentioned macros. In fig. 5 shows a screen with the settings made for these commands.

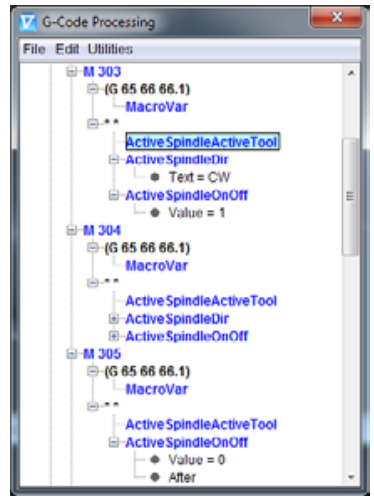

Fig. 5. Configuring commands M303, M304, M305 for drive tools

In cycles G87, G88 and G89 for machining holes perpendicular to the axis of the workpiece, the working movement is along the $\mathrm{X}$ axis, ie. in the YZ plane. In FIG. 6 shows a window for setting the G87 command. Vericur macro MotionPlaneYZ is used to set the working plane $\mathrm{YZ}$. The type of the preparatory command is read from the system variable for modal information \# 4009 [5].

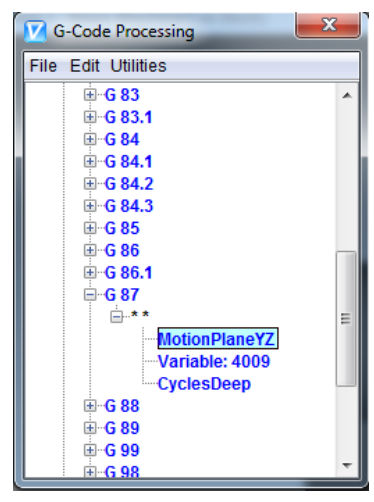

Fig. 6. Configuring G87 command

The type of the cycle is determined by Veicut macro CycleDeep. As already mentioned, in these cycles the working movement is on the $\mathrm{X}$-axis. By default, the working movement is performed on the Z-axis. For this purpose, the settings shown in fig. 7.

In order for the working movement to be on the $\mathrm{X}$-axis, the conditions are introduced:

\section{The type cycle is on (Cycle On);}

2. The coordinates in the type cycle must be set absolutely or incrementally (ABS_INC);
3. The preparatory command for the cycle is G87, G88 or G89.

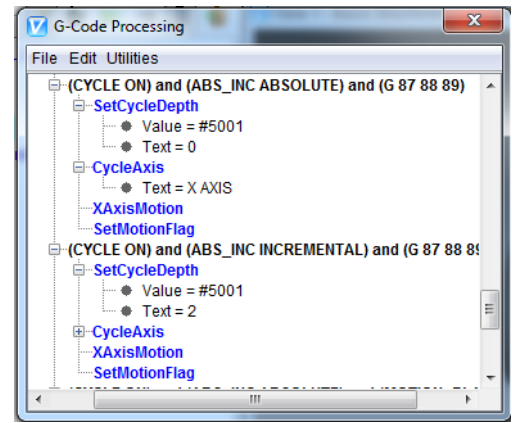

Fig. 7. Settings related to the output of the final coordinate along the $X$ axis when machining holes

If these conditions are met with the SetCycleDepth macro, it is specified that the hole depth will be determined by the system variable for position information \# 5001 . This is the variable in which the final $\mathrm{X}$-axis coordinate is stored relative to the current operating coordinate system. The Text field of the SetCycleDepth macro is set to 0 , which means that the value in the address is set to absolute. The CycleAxis macro determines the axis along which the machining is performed, depending on the selected working plane, in this case the $\mathrm{X}$ axis. The XAxisMotion macro indicates movement along the $\mathrm{X}$ axis. The G88 cycle settings are made in a similar way, using the CyclesTap macro.

\section{CONCLUSION}

1. The special M-commands used in the operation of the real machine Emco Maxxturn 45 and control Fanuc 18i TB are configured, using Vericut Macro.

2. Fixed cycles for machining radial holes are configured using Vericut Macro

\section{ACKNOWLEDGEMENTS}

The author would like to thank the Research and Development Sector at the Technical University of Sofia for the financial support.

\section{REFERENCES}

[1] www.cgtech.com

[2] www.webspring.ncsimul.com

[3] Kaldashev Ts., Developing a kinematic model for turning center with sub-spindle with Vericut, Youth Scientific Conference "Machines, Innovations, Technologies", November 8-9, 2018, "Bulgarian Journal of Engineering Design", pp. 35-40, ISSN 13137530, issue №37

[4] Vericut Help

[5] FANUC Operator's Manual Series 18i- MB

[6] Programming/Operation Fanuc 18i - Turning Maxxturn 45, PMCEmco, V490 